\title{
SPLITTING THE CONSERVATION PROCESS INTO CREATION AND ANNIHILATION PARTS
}

\author{
NICOLAS PRIVAULT \\ Equipe d'Analyse et Probabilités, Université d'Evry-Val d'Essonne \\ Boulevard des Coquibus, 91025 Evry Cedex, France \\ E-mail: privault@lami.univ-evry.fr
}

\begin{abstract}
The aim of this paper is the study of a non-commutative decomposition of the conservation process in quantum stochastic calculus. The probabilistic interpretation of this decomposition uses time changes, in contrast to the spatial shifts used in the interpretation of the creation and annihilation operators on Fock space.
\end{abstract}

1. Notation and preliminaries. Consider the Fock space $\Gamma(H)=\bigoplus_{n \geq 0} H^{\circ n}$ over a Hilbert space $H$, where $H^{\circ n}$ consists of the space of symmetric tensors in the tensor product $H^{\otimes n}$, endowed with the norm

$$
\|\cdot\|_{H^{\circ n}}^{2}=n !\|\cdot\|_{H^{\otimes n}}^{2}, \quad n \in \mathrm{N} .
$$

The Malliavin gradient $\nabla^{-}: \Gamma(H) \rightarrow \Gamma(H) \otimes H$ is defined by transformation of the tensor $f^{\circ n}, f \in H$, into $\nabla^{-} f^{\circ n}=n f^{\circ(n-1)} \otimes f, n \in \mathrm{N}$, and the Skorohod integral $\nabla^{+}: \Gamma(H) \otimes H \rightarrow \Gamma(H)$ satisfies $\nabla^{+} f^{\circ n} \otimes g=f^{\circ n} \circ g, n \in \mathrm{N}$. Those operators are extended by polarization, linearity and closability to their respective domains in $\Gamma(H)$, $\Gamma(H) \otimes H$. We let $\Phi=\Gamma\left(L^{2}\left(\mathbf{R}_{+}\right)\right)$and denote by $\langle\cdot, \cdot\rangle$ the scalar product on $\Phi$. We call $\mathcal{S}$ the set of elements of $\Phi$ whose chaos expansion is finite and involves only functions which are $\mathcal{C}^{1}$ with compact supports. The basic annihilation, creation and conservation operators $a_{h}^{-}, a_{h}^{+}, a_{h}^{\circ}$ are defined as

$$
a_{h}^{-} F=\left(\nabla^{-} F, h\right)_{H}, \quad a_{h}^{+} F=\nabla^{+}(F \otimes h), \quad a_{h}^{\circ} F=\nabla^{+}(h \nabla F), \quad F \in \mathcal{S}, h \in H .
$$

We also let $a_{t}^{\varepsilon}=a_{1_{[0, t]}^{-}}^{-}, \varepsilon=-,+, \circ$. The process $\left(a_{t}^{\circ}\right)_{t \in \mathbf{R}_{+}}$is called the conservation process, or gauge process. For $A \in \mathcal{B}\left(\mathbf{R}_{+}\right)$, let $\pi_{A}: L^{2}\left(\mathbf{R}_{+}\right) \rightarrow L^{2}\left(\mathbf{R}_{+}\right)$denote the projection operators defined as $\pi_{A} f=1_{A} f, f \in L^{2}\left(\mathbf{R}_{+}\right)$, and let $\pi_{t]}=\pi_{[0, t]}, \pi_{[t}=\pi_{[t, \infty[}$,

1991 Mathematics Subject Classification: 81S25, 60H07.

The paper is in final form and no version of it will be published elsewhere. 
$t \in \mathbf{R}_{+}$. The exponential vector $\xi(f), f \in L^{2}\left(\mathbf{R}_{+}\right)$, is defined as

$$
\xi(f)=\sum_{n \in \mathrm{N}} \frac{1}{n !} f^{\circ} \text {. }
$$

We say that $F \in \Phi$ is $\mathcal{F}_{A}$-measurable if $\Gamma\left(\pi_{A}\right) F=F, A \in \mathcal{B}\left(\mathbf{R}_{+}\right)$. Let $\mathcal{S}([a, b[), 0 \leq a<b$,

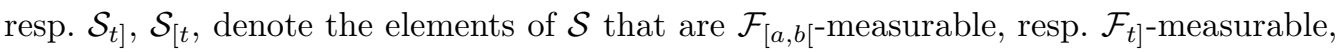
$\mathcal{F}_{[t}$-measurable. The second quantization $\Gamma(U): \Phi \rightarrow \Phi$ of a bounded operator $U$ : $L^{2}\left(\mathbf{R}_{+}\right) \rightarrow L^{2}\left(\mathbf{R}_{+}\right)$is defined as

$$
\Gamma(U)=\bigoplus_{n \geq 0} U^{\circ n}
$$

The Fock space $\Phi$ is isomorphic to the tensor product $\Phi_{t]} \otimes \Phi_{[t}, t \in \mathbf{R}_{+}$, via the mapping $\xi(f) \mapsto \xi\left(\pi_{t]} f\right) \otimes \xi\left(\pi_{[t} f\right)$, with $\Phi_{t]}=\Gamma\left(L^{2}([0, t])\right)$ and $\Phi_{t]}=\Gamma\left(L^{2}\left([t, \infty[)), t \in \mathbf{R}_{+}\right.\right.$, cf. [5]. In this paper, tensor products between vector spaces are completed if and only if vector spaces are complete.

2. A remark on non-commutative stochastic integration. The goal of this section is to notice that in quantum stochastic integration, integrator processes need not be adapted. Therefore, the stochastic integral of adapted processes can be defined with respect to processes other than $\left(a_{t}^{-}\right)_{t \in \mathrm{R}_{+}},\left(a_{t}^{+}\right)_{t \in \mathrm{R}_{+}}$and $\left(a_{t}^{\circ}\right)_{t \in \mathrm{R}_{+}}$. We recall that a process $\left(X_{t}\right)_{t \in \mathrm{R}_{+}}$of operators on $\Phi$ is said to be adapted if $X_{t}$ is written as $Y_{t} \otimes I_{d}$ on $\Phi_{t]} \otimes \Phi_{[t}$, $t \in \mathbf{R}_{+}$, where $I_{d}$ denotes the identity operator, cf. [1], [3]. If $U^{-}: \Phi \rightarrow \Phi \otimes L^{2}\left(\mathbf{R}_{+}\right)$ and $U^{+}: \Phi \otimes L^{2}\left(\mathbf{R}_{+}\right) \rightarrow \Phi$ are operators densely defined on $\mathcal{S}$ and $\mathcal{S} \otimes L^{2}\left(\mathbf{R}_{+}\right)$, we let $u_{h}^{-}, u_{h}^{+}: \Phi \rightarrow \Phi, h \in L^{2}\left(\mathbf{R}_{+}\right)$, be the operators defined on $\mathcal{S}$ as

$$
u_{h}^{-} F=\left(h, U^{-} F\right)_{L^{2}\left(\mathrm{R}_{+}\right)}, \quad u_{h}^{+} F=U^{+}(F \otimes h), F \in \mathcal{S}, \quad h \in L^{2}\left(\mathbf{R}_{+}\right) .
$$

We also let $u_{t}^{-}=u_{1_{[0, t]}^{-}}, u_{t}^{+}=u_{1_{[0, t]}^{+}}^{+}, t \in \mathbf{R}_{+}$, and note that $\left(u_{t}^{-}\right)_{t \in \mathrm{R}_{+}}$is adapted if and only if $\left(u_{t}^{+}\right)_{t \in \mathrm{R}_{+}}$is.

Definition 1. Let $\mathcal{A}$ denote the class of couples $\left(U^{-}, U^{+}\right)$where $U^{-}: \Phi \rightarrow \Phi \otimes$ $L^{2}\left(\mathbf{R}_{+}\right)$and $U^{+}: \Phi \otimes L^{2}\left(\mathbf{R}_{+}\right) \rightarrow \Phi$ are two closable and mutually adjoint operators densely defined on $\mathcal{S}$, that map $\mathcal{S}_{t]}$ into $\Phi_{t]}$ and $\mathcal{S}_{[t}$ into $\Phi_{[t}$, and such that

$$
u_{h}^{-}(F \otimes G)=\left(u_{h}^{-} F\right) \otimes G+F \otimes\left(u_{h}^{-} G\right), \quad F \in \mathcal{S}_{t]}, G \in \mathcal{S}_{[t}, \quad h \in L^{2}\left(\mathbf{R}_{+}\right), t \in \mathbf{R}_{+} .
$$

We note that $\left(\nabla^{-}, \nabla^{+}\right)$belongs to $\mathcal{A}$.

Definition 2. A couple $\left(U^{-}, U^{+}\right) \in \mathcal{A}$ is said to be an extension of the stochastic integral if $\Gamma\left(\pi_{s]}\right) U_{t}^{-}=\Gamma\left(\pi_{s]}\right) \nabla_{t}^{-}, \quad 0 \leq s<t, t \in \mathbf{R}_{+}$.

The last property states that the adapted projections of $U^{-} F$ and $\nabla F$ coincide $\forall F \in$ $\mathcal{S}$. Let $\mathcal{U}$ denote the set of elements of $\Phi \otimes L^{2}\left(\mathbf{R}_{+}\right)$of the form $\sum_{i=1}^{i=n} F_{i} \otimes h_{i}$, with $h_{1}, \ldots, h_{n} \in \mathcal{C}_{c}^{1}\left(\mathbf{R}_{+}\right)$, and $F_{1}, \ldots, F_{n} \in \mathcal{S}, n \in \mathrm{N}$. The set of square-integrable adapted processes is the completion in $\Phi \otimes L^{2}\left(\mathbf{R}_{+}\right)$of the set of adapted processes in $\mathcal{U}$. If $\Phi$ is identified to the $L^{2}$-space of a stochastic process $\left(Z_{t}\right)_{t \in \mathrm{R}_{+}}$with stationary independent increments and $\left(U^{-}, U^{+}\right) \in \mathcal{A}$ is an extension of the stochastic integral, then $U^{+}$coincides with the stochastic integral with respect to $\left(Z_{t}\right)_{t \in \mathrm{R}_{+}}$on the square-integrable adapted processes. 
Proposition 1. A couple $\left(U^{-}, U^{+}\right) \in \mathcal{A}$ is an extension of the stochastic integral if and only if $U^{+}$and $\nabla^{+}$coincide on the square-integrable adapted processes.

Pr oof. Let $1_{[a, b[} G \in \mathcal{U}$ be a simple adapted process. We have

$$
\begin{aligned}
\left\langle U^{+}\left(1_{[a, b[} G\right), F\right\rangle & =\int_{0}^{\infty} 1_{[a, b[}(s)\left\langle G, U_{s}^{-} F\right\rangle d s \\
& =\int_{0}^{\infty} 1_{[a, b[}(s)\left\langle G, \nabla_{s}^{-} F\right\rangle d s=\left\langle\nabla^{+}\left(1_{[a, b[} G\right), F\right\rangle, \quad F \in \mathcal{S} .
\end{aligned}
$$

This statement is extended by linearity and closability to the square-integrable adapted processes in $\Phi \otimes L^{2}\left(\mathbf{R}_{+}\right)$.

Remark 1 . Let $\left(U^{-}, U^{+}\right) \in \mathcal{A}$ be an extension of the stochastic integral, and assume that $\left(u_{t}^{-}\right)_{t \in \mathrm{R}_{+}},\left(u_{t}^{+}\right)_{t \in \mathrm{R}_{+}}$are adapted. Then $\left(U^{-}, U^{+}\right)=\left(\nabla^{-}, \nabla^{+}\right)$.

Proof. We will show that for any $t \in \mathbf{R}_{+}, \Gamma\left(\pi_{s]}\right) U_{t}^{-}=\Gamma\left(\pi_{s]}\right) \nabla_{t}^{-}, s<t$, and $\Gamma\left(\pi_{[s}\right) U_{t}^{-}=\Gamma\left(\pi_{[s}\right) \nabla_{t}^{-}, s>t$, on $\mathcal{S}$. The first part is a consequence of the fact that $\left(U^{-}, U^{+}\right)$is an extension of the stochastic integral. Assume $t<a<s$ and let $F \in \mathcal{S}_{a]}$, $G \in \mathcal{S}_{[a}$. Then since $\left(u_{t}^{-}\right)_{t \in \mathrm{R}_{+}}$and $\left(a_{t}^{-}\right)_{t \in \mathrm{R}_{+}}$are adapted,

$$
\begin{aligned}
\Gamma\left(\pi_{[s}\right) U_{t}^{-}(F \otimes G) & =\Gamma\left(\pi_{[s}\right)\left(\left(U_{t}^{-} F\right) \otimes G\right)=\left\langle U_{t}^{-} F, 1\right\rangle \Gamma\left(\pi_{[s}\right) G \\
& =\left\langle\nabla_{t}^{-} F, 1\right\rangle \Gamma\left(\pi_{[s}\right) G=\Gamma\left(\pi_{[s}\right) \nabla_{t}^{-}(F \otimes G),
\end{aligned}
$$

since $\Gamma\left(\pi_{[s}\right) U_{t}^{-} F=\left\langle U_{t}^{-} F, 1\right\rangle=\left\langle\nabla_{t}^{-} F, 1\right\rangle=\Gamma\left(\pi_{[s}\right) \nabla_{t}^{-} F, a<s$. Hence $U^{-}=\nabla^{-}$.

As a consequence of this remark, if $\left(U^{-}, U^{+}\right) \in \mathcal{A}$ is an extension of the stochastic integral that differs from $\left(\nabla^{-}, \nabla^{+}\right)$, then $\left(u_{t}^{-}\right)_{t \in \mathrm{R}_{+}}$and $\left(u_{t}^{+}\right)_{t \in \mathrm{R}_{+}}$cannot be adapted.

Remark 2. Let $\left(U^{-}, U^{+}\right) \in \mathcal{A}$. For the stochastic integral of simple adapted processes to be defined with respect to $d u_{t}^{-}, d u_{t}^{+}$, it is sufficient that

$$
\left(u_{t}-u_{s}\right)=I_{d} \otimes\left(u_{t}-u_{s}\right) \text { on } \Phi_{s]} \otimes \Phi_{[s}, \forall s \geq t, \quad t \in \mathbf{R}_{+} .
$$

This condition is satisfied in particular if $\left(U^{-}, U^{+}\right)$is an extension of the stochastic integral. Hence quantum stochastic integrals of simple adapted operator processes $\left(u_{t}^{-}\right)_{t \in \mathrm{R}_{+}}$, $\left(u_{t}^{+}\right)_{t \in \mathrm{R}_{+}}$can be defined without requiring the adaptedness of $\left(u_{t}^{-}\right)_{t \in \mathrm{R}_{+}},\left(u_{t}^{+}\right)_{t \in \mathrm{R}_{+}}$.

Proof. Let

$$
X=\sum_{i=1}^{i=n} X_{i} 1_{\left[t_{i}, t_{i+1}[\right.}
$$

be a simple adapted operator process. The composition $\left(u_{t}^{-}-u_{s}^{-}\right) X_{s}, t \geq s$, is well-defined by

$$
\left(u_{t}^{-}-u_{s}^{-}\right) X_{s}(F \otimes G)=X_{s} F \otimes\left(u_{t}^{-}-u_{s}^{-}\right) G, \quad t \geq s
$$

on $\Phi_{s]} \otimes \Phi_{[s}$. Consequently, the integral of $X$ with respect to $d u_{t}^{-}$is well-defined as

$$
\int_{0}^{\infty} X_{s} d u_{s}^{-}=\sum_{i=1}^{i=n} X_{i}\left(u_{t_{i+1}}^{-}-u_{t_{i}}^{-}\right) .
$$


And $\int_{0}^{\infty} X_{s} d u_{s}^{+}$is defined by duality as

with

$$
\int_{0}^{\infty} X_{s} d u_{s}^{+}=\sum_{i=1}^{i=n} X_{i}\left(u_{t_{i+1}}^{+}-u_{t_{i}}^{+}\right)
$$

We may also write

$$
\left\langle\int_{0}^{\infty} X_{s} d u_{s}^{-} F, G\right\rangle=\left\langle F, \int_{0}^{\infty} X_{s}^{*} d u_{s}^{+} G\right\rangle, \quad F, G \in \mathcal{S} .
$$

$$
\int_{0}^{\infty} X_{s} d u_{s}^{-} F=\int_{0}^{\infty} U_{s}^{-} X_{s} F d s, \quad \int_{0}^{\infty} X_{s} d u_{s}^{+} F=U^{+}(X F), \quad F \in \mathcal{S} .
$$

The notion of extension of the stochastic integral on Fock space also allows to extend the Clark representation formula, cf. $[2,8]$.

Proposition 2. Let $\left(U^{-}, U^{+}\right) \in \mathcal{A}$ be an extension of the stochastic integral on Fock space. Then the mapping $F \mapsto\left(\Gamma\left(\pi_{s]}\right) U_{s}^{-} F\right)_{s \in \mathrm{R}_{+}}$is continuous from $\Phi$ to $\Phi \otimes L^{2}\left(\mathbf{R}_{+}\right)$, and any $F \in \Phi$ can be represented as

$$
F=\langle F, 1\rangle+U^{+}\left(\Gamma\left(\pi_{\cdot]}\right) U^{-} F\right) .
$$

Proof. For $F \in \mathcal{S}, \Gamma\left(\pi_{t]}\right) U_{t}^{-} F$ is defined as

$$
\Gamma\left(\pi_{t]}\right) U_{t}^{-} F=\lim _{s \uparrow t} \Gamma\left(\pi_{s]}\right) U_{t}^{-} F=\lim _{s \uparrow t} \Gamma\left(\pi_{s]}\right) \nabla_{t}^{-} F=\Gamma\left(\pi_{t]}\right) \nabla_{t}^{-} F, \quad t \in \mathbf{R}_{+} .
$$

The continuity result and the representation formula are well-known facts in case $\left(U^{-}, U^{+}\right)=\left(\nabla^{-}, \nabla^{+}\right)$, cf. [12], and they immediately extend to $U^{-}$from its definition and Proposition 1.

3. A decomposition of the conservation operator. The main goal of this section is to introduce a non-commutative decomposition of the number operator on Fock space and study its probabilistic interpretations. Let $\stackrel{\circ}{h}$, denote the function defined as $\stackrel{\circ}{h}(t)=$ $\int_{0}^{t} h(s) d s, h \in L^{2}\left(\mathbf{R}_{+}\right)$, and let $f^{\prime}(t)=\frac{d}{d t} f(t), t \in \mathbf{R}_{+}, f \in \mathcal{C}_{c}^{1}\left(\mathbf{R}_{+}\right)$.

Definition 3. We define the linear operators $\nabla^{\ominus}: \Phi \rightarrow \Phi \otimes L^{2}\left(\mathbf{R}_{+}\right)$on $\mathcal{S}$ and $\nabla^{\oplus}: \Phi \otimes L^{2}\left(\mathbf{R}_{+}\right) \rightarrow \Phi$ on $\mathcal{U}$ by

$$
\nabla_{t}^{\ominus} f^{\circ n}=-n\left(\pi_{[t} f^{\prime}\right) \circ f^{\circ(n-1)}, \quad t \in \mathbf{R}_{+}, f \in \mathcal{C}_{c}^{1}\left(\mathbf{R}_{+}\right), \quad n \in \mathbf{N},
$$

and

and by polarization of these expressions.

$$
\nabla^{\oplus}\left(f^{\circ n} \otimes h\right)=n(f \stackrel{\circ}{h})^{\prime} \circ f^{\circ(n-1)}, \quad f, h \in \mathcal{C}_{c}^{1}\left(\mathbf{R}_{+}\right),
$$

The operators $\nabla^{\ominus}$ and $\nabla^{\oplus}$ are unbounded, closable, densely defined, and adjoint of each other. We note that $\left(\nabla^{-}+\nabla^{\ominus}, \nabla^{+}+\nabla^{\oplus}\right) \in \mathcal{A}$ and is an extension of the stochastic integral. Let also $a_{h}^{\ominus}, a_{h}^{\oplus}$ be defined on $\mathcal{S}$ as

$$
a_{h}^{\ominus} F=\left(\nabla^{\ominus} F, h\right)_{L^{2}\left(\mathrm{R}_{+}\right)}, \quad a_{h}^{\oplus} F=\nabla^{\oplus}(F \otimes h), \quad n \in \mathrm{N}, \quad F \in \mathcal{S}, \quad h \in L^{2}\left(\mathbf{R}_{+}\right),
$$

and let $a_{t}^{\ominus}=a_{1_{[0, t]}}^{\ominus}, a_{t}^{\oplus}=a_{1_{[0, t]}}^{\oplus}, t \in \mathbf{R}_{+}$. The operator $a_{h}^{\ominus}$ is adjoint to $a_{h}^{\oplus}, h \in L^{2}\left(\mathbf{R}_{+}\right)$. Moreover, $a_{h}^{\ominus}$ and $a_{h}^{\oplus}$ can be defined as the differential second quantizations of $f \mapsto \stackrel{\circ}{h} f^{\prime}$ 
and $f \mapsto(f \stackrel{\circ}{h})^{\prime}$ as

$$
a_{h}^{\ominus}=d \Gamma(\stackrel{\circ}{h} \partial), \quad a_{h}^{\oplus}=d \Gamma(\partial \stackrel{\circ}{h}) .
$$

The following proposition is a simple consequence of the derivation rule of the product of functions of real variable.

Proposition 3. The operator $a_{h}^{\circ}$ admits the non-commutative decomposition

$$
a_{h}^{\circ}=a_{h}^{\ominus}+a_{h}^{\oplus}, \quad h \in L^{2}\left(\mathbf{R}_{+}\right),
$$

into the sum of two adjoint operators.

The operator $a_{h}^{\ominus}+a_{h}^{\oplus}$ has a Poisson law with parameter $\|h\|_{L^{2}\left(\mathrm{R}_{+}\right)}^{2}$ in the state $\xi(h) \exp \left(-\frac{1}{2}\|h\|_{L^{2}\left(\mathrm{R}_{+}\right)}^{2}\right)$, and $\left(a_{t}^{\ominus}+a_{t}^{\oplus}\right)_{t \in \mathrm{R}_{+}}$is a non-commutative Poisson process in this state, cf. e.g. [1].

Proposition 4. We have the commutation relations

$$
\left[\nabla_{s}^{-}, \nabla_{t}^{\ominus}\right] \xi(f)=-\left(\pi_{[t} f^{\prime}\right)(s) \xi(f), \quad\left[a_{h}^{+}, \nabla_{t}^{\ominus}\right] \xi(f)=\left(\pi_{[t} h^{\prime}\right) \circ \xi(f),
$$

$f, h \in \mathcal{C}_{c}^{1}\left(\mathbf{R}_{+}\right), s, t \in \mathbf{R}_{+}$.

Proof. We have $\nabla_{s}^{-} \nabla_{t}^{\ominus} f^{\circ n}=-n\left(\pi_{[t} f^{\prime}\right)(s) f^{\circ(n-1)}-n(n-1) f(s)\left(\pi_{[t} f^{\prime}\right) \circ f^{\circ(n-2)}$, and $\nabla_{t}^{\ominus} \nabla_{s}^{-} f^{\circ n}=-n(n-1) f(s)\left(\pi_{[t} f^{\prime}\right) \circ f^{\circ(n-2)}$. On the other hand, $a_{h}^{+} \nabla_{t}^{\ominus} f^{\circ n}=$ $-n a_{h}^{+}\left(\pi_{[t} f^{\prime}\right) \circ f^{\circ(n-1)}$, and $\nabla_{t}^{\ominus} a_{h}^{+} f^{\circ n}=\nabla_{t}^{\ominus} h \circ f^{\circ n}=-n\left(\pi_{[t} f^{\prime}\right) \circ f^{\circ(n-1)} \circ h-\left(\pi_{[t} h^{\prime}\right) \circ f^{\circ n}$.

\section{Probabilistic interpretations of the decomposition}

4.1. Wiener space interpretation. Let $\left(W, L^{2}\left(\mathbf{R}_{+}\right), \mu\right)$ denote the classical Wiener space, with Brownian motion $\left(B_{t}\right)_{t \in \mathrm{R}_{+}}$. We work here under the identification of $L^{2}(W, \mu)$ and $\Phi$ provided by the multiple stochastic integrals with respect to $\left(B_{t}\right)_{t \in \mathrm{R}_{+}}$. We are interested here in the properties of $\nabla^{\ominus}$ under the above identification. Recall that on the Wiener space, $\nabla^{-}$is identified to a derivation operator which satisfies

$$
\left(\nabla^{-} F, h\right)_{L^{2}\left(\mathrm{R}_{+}\right)}=\lim _{\varepsilon \rightarrow 0} \frac{F\left(B .+\int_{0}^{\cdot} h(s) d s\right)-F}{\varepsilon}, \quad F \in \mathcal{S}, h \in L^{2}\left(\mathbf{R}_{+}\right),
$$

cf. e.g. [6]. For $h \in L^{2}\left(\mathbf{R}_{+}\right)$, with $\sup _{x \in \mathbf{R}_{+}}|h(x)|<1$, let $\nu_{h}(t)=t+\stackrel{\circ}{h}, t \in \mathbf{R}_{+}$.

Definition 4 . We define a mapping $\mathcal{T}_{h}: W \rightarrow W, t, \varepsilon \in \mathbf{R}_{+}$, as

$$
\mathcal{T}_{h}(\omega)=\omega \circ \nu_{h}^{-1}, \quad h \in L^{2}\left(\mathbf{R}_{+}\right), \sup _{x \in \mathbf{R}_{+}}|h(x)|<1 .
$$

The transformation $\mathcal{T}_{h}$ acts on the trajectory of $\left(B_{s}\right)_{s \in \mathrm{R}_{+}}$by change of time, or by perturbation of its predictable quadratic variation. Although $\mathcal{T}_{h}$ is not absolutely continuous with respect to the Wiener measure, the functional $F \circ \mathcal{T}_{h}$ is well-defined for $F \in \mathcal{S}$, since elements of $\mathcal{S}$ can be defined for every trajectory of $\left(B_{t}\right)_{t \in \mathrm{R}_{+}}$, using the product formula for multiple stochastic integrals and the relation $\int_{0}^{\infty} f_{s} d B_{s}=-\int_{0}^{\infty} f^{\prime}(s) B_{s} d s$, a.s., $f \in \mathcal{C}_{c}^{1}\left(\mathbf{R}_{+}\right)$.

Proposition 5 ([9]). We have for $F \in \mathcal{S}$

$$
\int_{0}^{\infty} h_{t}\left(\nabla_{t}^{\ominus}+\frac{1}{2} \nabla_{t}^{-} \nabla_{t}^{-}\right) F d t=-\lim _{\varepsilon \rightarrow 0} \frac{1}{\varepsilon}\left(F \circ \mathcal{T}_{\varepsilon h}-F\right) .
$$


4.2. Poisson space interpretation. We now consider a standard Poisson process on a probability space $(B, P)$. The Poisson multiple stochastic integrals provide an isometric isomorphism between $L^{2}(B)$ and $\Phi$, cf. [11]. From [7], $\nabla^{-}$satisfies

$$
\nabla_{t}^{-} f\left(T_{1}, \ldots, T_{n}\right)=\sum_{k=1}^{k=n} 1_{\left[T_{k-1}, T_{k}\right.}(t)\left(f\left(T_{1}, \ldots, T_{k}, t, T_{k+1}, \ldots, T_{n-1}\right)-f\left(T_{1}, \ldots, T_{n}\right)\right)
$$

$t \in \mathbf{R}_{+}, f \in \mathcal{C}_{c}^{1}\left(\mathbf{R}_{+}^{n+1}\right)$, which amounts to perturbing the trajectories of $\left(N_{t}\right)_{t \in \mathrm{R}_{+}}$by addition of a jump at time $t$. The operator $\nabla^{+}$coincides with the Poisson stochastic integral on the square-integrable adapted processes. The operator $\nabla_{t}^{-}+\nabla_{t}^{\ominus}$ on Poisson space, as $\nabla_{t}^{\ominus}+\frac{1}{2} \nabla_{t}^{-} \nabla_{t}^{-}$on the Wiener space, is defined by an infinitesimal time change on the Poisson process.

Proposition 6 ([9]). We have

$$
\left(\left(\nabla^{\ominus}+\nabla^{-}\right) F, h\right)_{L^{2}\left(\mathrm{R}_{+}\right)}=-\lim _{\varepsilon \rightarrow 0} \frac{1}{\varepsilon}\left(F \circ \mathcal{T}_{\varepsilon h}-F\right), \quad F \in \mathcal{S},
$$

where the transformation $\mathcal{T}_{h}$ is defined as in Def. 4, i.e. it acts on the Poisson process trajectories via the time change $\nu_{h}$.

Remark 3. Relations (2) and (4) imply, both in the Wiener and Poisson interpretations, the following product rule for $\nabla^{\ominus}$ :

$$
\nabla^{\ominus}(F G)=F \nabla^{\ominus} G+G \nabla^{\ominus} F-\nabla^{-} F \nabla^{-} G, \quad F, G \in \mathcal{S} .
$$

As a consequence of this remark, a duality argument shows that Relation (1) can be extended to random $h \in \mathcal{U}$ as:

$$
a_{h}^{\circ}+\nabla^{\oplus}(h)=a_{h}^{\ominus}+a_{h}^{\oplus}, \quad h \in L^{2}\left(\mathbf{R}_{+}\right),
$$

where $\nabla^{\oplus}(h)$ is identified to a multiplication operator, in both the Wiener and Poisson interpretations of $\Phi$. It is easy to check that $\nabla^{\oplus}(h)=0$ if $h$ is adapted, since $\left(\nabla^{-}+\right.$ $\left.\nabla^{\ominus}, \nabla^{+}+\nabla^{\oplus}\right) \in \mathcal{A}$ is an extension of the stochastic integral.

5. Non-commutative stochastic integration and Itô calculus. From Section 3, the integral of simple adapted processes with respect to the operator processes $\left(a_{t}^{\ominus}\right)_{t \in \mathrm{R}_{+}}$ and $\left(a_{t}^{\oplus}\right)_{t \in \mathbf{R}_{+}}$are well-defined. However, the processes $\left(a_{t}^{\ominus}\right)_{t \in \mathbf{R}_{+}}$and $\left(a_{t}^{\oplus}\right)_{t \in \mathbf{R}_{+}}$are not adapted, hence iterated stochastic integrals of simple adapted processes with respect to $\left(a_{t}^{\varepsilon}\right)_{t \in \mathrm{R}_{+}}, \varepsilon=\ominus, \oplus$ have to be defined as anticipating stochastic integrals, and $a_{t}^{\ominus}, a_{t}^{\oplus}$ do not commute in general with the differential $d u_{t}^{ \pm}$. Our construction of anticipating quantum stochastic integration parallels that of [4] but concerns $\left(a_{t}^{\ominus}\right)_{t \in \mathrm{R}_{+}}$and $\left(a_{t}^{\oplus}\right)_{t \in \mathrm{R}_{+}}$. For $U^{-}=\nabla^{-}, \nabla^{\ominus}$, the integrals $\int_{0}^{t} d u_{s}^{-} a_{s}^{\varepsilon}, \int_{0}^{t} a_{s}^{\varepsilon} d u_{s}^{+}, \varepsilon=\ominus, \oplus$, are defined on $\mathcal{S}$ as

$$
\int_{0}^{t} d u_{s}^{-} a_{s}^{\varepsilon} F=\int_{0}^{t} U_{s}^{-} a_{s}^{\varepsilon} F, \quad \int_{0}^{t} d u_{s}^{+} a_{s}^{\varepsilon} F=U^{+}\left(1_{[0, t]}(\cdot) a^{\varepsilon} F\right), \quad F \in \mathcal{S} .
$$

The integrals $\int_{0}^{t} a_{s}^{\varepsilon} d u_{s}^{+}, \int_{0}^{t} a_{s}^{\varepsilon} d u_{s}^{-}$are defined by duality from $\int_{0}^{t} d u_{s}^{-} a_{s}^{\varepsilon}, \int_{0}^{t} d u_{s}^{+} a_{s}^{* \varepsilon}$, with $* \varepsilon=\oplus, \ominus$ respectively if $\varepsilon=\ominus, \oplus$. 
Proposition 7. Let $\left(U^{-}, U^{+}\right) \in \mathcal{A}$ and $\left(V^{-}, V^{+}\right) \in \mathcal{A}$ be extensions of the stochastic integral. We have the multiplication table

\begin{tabular}{|c|c|c|c|}
\hline$\cdot$ & $d t$ & $d u_{t}^{-}$ & $d v_{t}^{+}$ \\
\hline$d t$ & 0 & 0 & 0 \\
\hline$d v_{t}^{+}$ & 0 & 0 & 0 \\
\hline$d u_{t}^{-}$ & 0 & 0 & $d u_{t}^{-} \cdot d v_{t}^{+}$ \\
\hline
\end{tabular}

The non-vanishing product $d u_{t}^{-} \cdot d v_{t}^{+}$is given as

$$
\begin{gathered}
\left(d a_{t}^{\ominus}+d a_{t}^{\oplus}\right) \cdot\left(d a_{t}^{\ominus}+d a_{t}^{\oplus}\right)=d a_{t}^{\circ}, \quad d a_{t}^{-} \cdot\left(d a_{t}^{+}+d a_{t}^{\oplus}\right)=d t+d a_{t}^{-}, \\
\left(d a_{t}^{-}+d a_{t}^{\ominus}\right) \cdot d a_{t}^{+}=d t+d a_{t}^{+}, \quad d a_{t}^{-} \cdot d a_{t}^{+}=d t,
\end{gathered}
$$

respectively if $\left(U^{-}, V^{+}\right)=\left(\nabla^{-}+\nabla^{\ominus}, \nabla^{+}+\nabla^{\oplus}\right),\left(U^{-}, V^{+}\right)=\left(\nabla^{-}, \nabla^{+}+\nabla^{\oplus}\right),\left(U^{-}, V^{+}\right)$ $=\left(\nabla^{-}+\nabla^{\ominus}, \nabla^{+}\right),\left(U^{-}, V^{+}\right)=\left(\nabla^{-}, \nabla^{+}\right)$.

Pr o of. The following relations hold:

$$
\begin{aligned}
\left\langle u_{t}^{+} F, v_{t}^{-} G\right\rangle= & \int_{0}^{t}\left\langle u_{s}^{+} F, V_{s}^{-} G\right\rangle d s+\left\langle F, \int_{0}^{t} U_{s}^{-} v_{s}^{-} G d s\right\rangle, \\
\left\langle v_{t}^{-} G, u_{t}^{-} F\right\rangle= & \int_{0}^{t s}\left\langle V_{0}^{t}\left\langle V_{z}^{-} G, U_{s}^{-} F\right\rangle d z d s+\int_{0}^{u} \int_{0}\left\langle V_{z}^{-} G, U_{s}^{-} F\right\rangle d z d s,\right.
\end{aligned}
$$

$F, G \in \mathcal{S}$. Let $X, Y$ be simple adapted processes such that $\mathcal{S} \subset \operatorname{Dom}\left(X_{s} Y_{s}\right), s \in \mathbf{R}_{+}$. By linearity and adaptedness of $\left(X_{t}\right)_{t \in \mathrm{R}_{+}},\left(Y_{t}\right)_{t \in \mathrm{R}_{+}},(6)$ implies

$$
\begin{aligned}
& \left\langle\int_{0}^{t} Y_{s} d v_{s}^{+} G, \int_{0}^{t} X_{s}^{*} d u_{s}^{-} F\right\rangle \\
& =\int_{0}^{t}\left\langle\int_{0}^{s} Y_{z} d v_{z}^{+} G, X_{s}^{*} U_{s}^{-} F\right\rangle d s+\int_{0}^{t}\left\langle Y_{s} G, V_{s}^{-} \int_{0}^{s} X_{z}^{*} d u_{z}^{-} F\right\rangle d s,
\end{aligned}
$$

and by duality,

$$
\begin{aligned}
& \left\langle\int_{0}^{t} Y_{s} d u_{s}^{-} G, \int_{0}^{t} X_{s}^{*} d v_{s}^{+} F\right\rangle \\
& =\int_{0}^{t}\left\langle U_{s}^{-} G, Y_{s}^{*} \int_{0}^{s} X_{z}^{*} d v_{z}^{+} F\right\rangle d s+\int_{0}^{t}\left\langle V_{s}^{-} X_{s} \int_{0}^{s} Y_{z} d u_{z}^{-} G, F\right\rangle d s,
\end{aligned}
$$

for $F, G \in \mathcal{S}$. Those relations are written as

$$
\begin{aligned}
& \int_{0}^{t} X_{s} d u_{s}^{+} \int_{0}^{t} Y_{s} d v_{s}^{+}=\int_{0}^{t} d u_{s}^{+} X_{s}\left(\int_{0}^{s} Y_{z} d v_{z}^{+}\right)+\int_{0}^{t}\left(\int_{0}^{s} X_{z} d u_{z}^{+}\right) Y_{s} d v_{s}^{+}, \\
& \int_{0}^{t} X_{s} d v_{s}^{-} \int_{0}^{t} Y_{s} d u_{s}^{-}=\int_{0}^{t}\left(\int_{0}^{s} X_{z} d v_{z}^{-}\right) Y_{s} d u_{s}^{-}+\int_{0}^{s} d v_{s}^{-} X_{s}\left(\int_{0}^{s} Y_{z} d u_{z}^{-}\right),
\end{aligned}
$$

hence $d u_{t}^{+} \cdot d v_{t}^{+}=0$, and $d u_{t}^{-} \cdot d v_{t}^{-}=0$. We have from $(7)$ 


$$
\left\langle\int_{0}^{t} Y_{s} d v_{s}^{-} G, \int_{0}^{t} X_{s}^{*} d u_{s}^{-} F\right\rangle=\int_{0}^{t}\left\langle\int_{0}^{s} Y_{z} d v_{z}^{-} G, X_{s}^{*} U_{s}^{-} F\right\rangle d s+\int_{0}^{t}\left\langle Y_{s} V_{s}^{-} G, \int_{0}^{s} X_{z}^{*} d u_{z}^{-} F\right\rangle d s,
$$

hence

$$
\int_{0}^{t} X_{s} d u_{s}^{+} \int_{0}^{t} Y_{s} d v_{s}^{-}=\int_{0}^{t} d u_{s}^{+} X_{s}\left(\int_{0}^{s} Y_{z} d v_{z}^{-}\right)+\int_{0}^{t}\left(\int_{0}^{s} X_{z} d u_{z}^{+}\right) Y_{s} d v_{s}^{-},
$$

which means that $d u_{t}^{+} \cdot d v_{t}^{-}=0$. The non-zero terms are explicitly computed in [10]. We notice here that they can also be obtained formally from the well-known relations $d\left(a_{t}^{\ominus}+a_{t}^{\oplus}\right) \cdot d\left(a_{t}^{\ominus}+a_{t}^{\oplus}\right)=d a_{t}^{\ominus}+d a_{t}^{\oplus}, \quad d a_{t}^{-} \cdot d\left(a_{t}^{\ominus}+a_{t}^{\oplus}\right)=d a_{t}^{-}, \quad d\left(a_{t}^{\ominus}+a_{t}^{\oplus}\right) \cdot d a_{t}^{+}=d a_{t}^{+}$.

The Itô multiplication table now gives the commutation relations

$$
d\left[a_{t}^{\ominus}, a_{t}^{+}\right]=d a_{t}^{-}+\left[a_{t}^{\ominus}, d a_{t}^{+}\right], \quad d\left[a_{t}^{-}, a_{t}^{\oplus}\right]=d a_{t}^{+}+\left[d a_{t}^{-}, a_{t}^{\oplus}\right],
$$

and

$$
d\left[a_{t}^{\ominus}, a_{t}^{\oplus}\right]=d a_{t}^{\circ}+\left[a_{t}^{\ominus}, d a_{t}^{\oplus}\right]+\left[d a_{t}^{\ominus}, a_{t}^{\oplus}\right] .
$$

\section{References}

[1] P. Biane, Calcul stochastique non-commutatif, in: Ecole d'été de Probabilités de SaintFlour, volume 1608 of Lecture Notes in Mathematics, Saint-Flour, 1993. Springer-Verlag.

[2] J. M. C. Clark, The representation of functionals of Brownian motion by stochastic integrals, Ann. Math. Stat. 41 (1970), 1281-1295.

[3] R. L. Hudson and K. R. Parthasarathy, Quantum Itô's formula and stochastic evolutions, Commun. Math. Phys. 93 (1984), 301-323.

[4] J. M. Lindsay, Quantum and non-causal stochastic calculus, Probab. Theory Related Fields 97 (1993), 65-80.

[5] P. A. Meyer, Quantum Probability for Probabilists, volume 1538 of Lecture Notes in Mathematics, Springer-Verlag, Berlin/New York 1993.

[6] D. Nualart, The Malliavin Calculus and Related Topics, Probability and its Applications, Springer-Verlag, Berlin/New York 1995.

[7] D. Nualart and J. Vives, Anticipative calculus for the Poisson process based on the Fock space, in: J. Azéma, P.A. Meyer, and M. Yor (eds.), Séminaire de Probabilités XXIV, volume 1426 of Lecture Notes in Mathematics, pp. 154-165. Springer-Verlag, Berlin/New York 1990.

[8] D. Ocone, A guide to the stochastic calculus of variations, in: H. Körezlioğlu and A.S. Üstünel (eds.), Stochastic Analysis and Related Topics, Silivri, 1988; volume 1316 of Lecture Notes in Mathematics, Springer-Verlag, Berlin/New York 1988.

[9] N. Privault, A calculus on Fock space and its probabilistic interpretations, Bull. Sci. Math., to appear.

[10] N. Privault, An extension of the quantum Itô table and its matrix representation, to appear in Quantum Probability Communications X, World Scientific, 1998.

[11] D. Surgailis, On multiple Poisson stochastic integrals and associated Markov semigroups, Probab. Math. Stat. 3 (1984), 217-239.

[12] A. S. Üstünel, Representation of the distributions on Wiener space and stochastic calculus of variations, J. Funct. Anal. 70 (1987), 126-129. 